\title{
Green's function retrieval in a field of random water waves
}

\author{
Michael G. Brown*, Chuntao Lu \\ Rosenstiel School of Marine and Atmospheric Sciences, University of Miami \\ 4600 Rickenbacker Cswy., Miami FL 33149 USA
}

\begin{abstract}
It has recently been demonstrated using a variety of wave types that crosscorrelating time series of apparently random waves measured at two locations yields an estimate of the Green's function that describes the wave field generated at one of those locations and measured at the other. This procedure can be described as random wave interferometry. In this paper random surface gravity wave interferometry is described theoretically, demonstrated using numerical simulations, and investigated experimentally using both wavetank measurements and ocean wave measurements. Simulations and wavetank measurements are in good agreement with theoretical predictions, but the oceanmeasurement-based cross correlations do not yield the predicted structure. Possible explanations are discussed.
\end{abstract}

Keywords: Green's function retrieval, random wave interferometry, random wave field cross-correlations, water waves

2010 MSC: 76B15, 62H20

\section{Introduction}

In recent years it has become appreciated that deterministic wave propagation information can be extracted from an apparently random wave field via a process that can be described as random wave interferometry; the cross-

${ }^{*}$ Corresponding author, Email address: mbrown@rsmas.miami.edu

Preprint submitted to Wave Motion

July 26, 2015

(C) 2015. This manuscript version is made available under the Elsevier user license http://www.elsevier.com/open-access/userlicense/1.0/ 
correlation of measurements at two locations of a random wave field yields an approximation to the Green's function that describes propagation between those locations $[1,2,3,4,5,6,17,8,9,10,11,12,13$. Random wave interferometry has been widely investigated in the context of elastic waves in solids [14, 15, 16], including seismic[17, 18, 19, 20, 21, 22] and helioseismic 23, 24] applications, and sound waves in fluids, including applications to ocean acoustics [25, 26, 27, 28, 29, 30, 31, 32, 33, 34, 35] and atmospheric acoustics 36, 37, 38. The underlying theory is widely applicable, as it can be applied to any type of linear wave propagation.

In this paper we study, both theoretically and experimentally, the application of these ideas to water waves, i.e., surface gravity waves. This topic can be referred to as random water wave interferometry. Previous work on random water wave interferometry is described in [39] and [40. 39] focuses on theory relating to tsunamis (very low frequency ocean surface gravity waves for which $k h<<1$ ), while [40] presents theory and data analysis relating to infragravity waves (low frequency ocean surface gravity waves for which $k h=O(1)$ in the deep ocean). The latter work builds on the earlier work [41] which demonstrated that seafloor measurements of infragravity-wave-induced pressure fluctuations separated by a few 10's of km are coherent, and the work reported in [42], which demonstrated the utility of cross-correlating measurements of that type.

The basic result on which we focus in this paper is

$$
\gamma C_{A B}(t)=D(t) *\left[G_{\eta}\left(\mathbf{x}_{B} \mid \mathbf{x}_{A}, t\right)+G_{\eta}\left(\mathbf{x}_{A} \mid \mathbf{x}_{B},-t\right)\right]
$$

Here $\gamma$ is a constant, $C_{A B}(t)$ is the correlation function of records of surface elevation $\eta(t)$ at locations $\mathbf{x}_{A}$ and $\mathbf{x}_{B}, D(t)$ is a band-limited approximation to a delta function, and $G_{\eta}\left(\mathbf{x} \mid \mathbf{x}_{0}, t\right)$ is the transient surface elevation at point $\mathbf{x}$ due to an impulsive point source at $\mathbf{x}_{0}$. Two derivations of Eq. (1), which holds 30 in both open and closed systems, are given below. It is important to appreciate that two results underlie Eq. (1) - an exact identity involving Green's functions, and a strong approximate statistical assumption. The Green's function identity holds provided the underlying fluid dynamical assumptions (adequacy 
of linear theory, including the assumption that the flow remains irrotational)

35 are satisfied. The statistical assumptions underlying Eq. (1) are strong and might reasonably be questioned, but we note that experience in other fields has shown that relationships similar to Eq. (1) are good approximations even when the underlying statistical assumptions are only approximately satisfied.

The remainder of this paper is organized as follows. The theory underlying

40 Eq. (1) is presented in section 2. Derivations of Eq. (1) for both open and closed systems are included. In section 3 simulations, in both open and closed systems, are presented and shown to be consistent with Eq. (1). In section 4 the analysis of data collected in a wavetank for the purpose of testing Eq. (1) is described. Agreement between theory and measurements is good. In section

455 the analysis of a set of ocean wave measurements, again to test Eq. (1), is described. Those results are not in good agreement with theory. In section 6 our results are discussed and summarized.

\section{Theory}

In this section we present two derivations of Eq. (1), which holds in both open and closed systems. Each derivation makes use of an identity involving Green's functions, Eq. (12) or (22), and a strong statistical assumption, Eq. (14) or (24).

\subsection{Preliminaries}

We begin with comments on two topics. First, it is important to distinguish between two different Green's functions: $G_{\phi}$ is the velocity potential Green's function evaluated at the undisturbed free surface; and $G_{\eta}$ is the surface displacement Green's function. Both of these quantities have time- and frequency-domain representations. Second, convolutions and cross-correlations play important roles in the derivations below. Consider real-valued functions

${ }_{60} f_{1}(t)$ and $f_{2}(t)$. Convolution of $f_{1}(t)$ and $f_{2}(t)$ is defined as $f_{1}(t) * f_{2}(t)=$ $\int_{-\infty}^{\infty} d \tau f_{1}(\tau) f_{2}(t-\tau)$. The cross-correlation of $f_{1}(t)$ and $f_{2}(t)$ is defined as 
$f_{1}(t) * f_{2}(-t)=\int_{-\infty}^{\infty} d \tau f_{1}(\tau) f_{2}(t+\tau)$. Let $\bar{f}(\omega)=F[f(t)]=\int_{-\infty}^{\infty} f(t) e^{i \omega t} d t$ denote the Fourier transform of $f(t)$, and let $f(t)=F^{-1}[\bar{f}(\omega)]$ denote the inverse transform. Because these variables are real-valued, it is assumed that ${ }_{65} \bar{f}(-\omega)=\bar{f}^{*}(\omega)$, where the superscript ${ }^{*}$ denotes complex conjugation. The Fourier transform of the convolution $f_{1}(t) * f_{2}(t)$ is $\bar{f}_{1}(\omega) \bar{f}_{2}(\omega)$, and the Fourier transform of the cross-correlation $f_{1}(t) * f_{2}(-t)$ is $\bar{f}_{1}(\omega) \bar{f}_{2}{ }^{*}(\omega)$. For the purpose of computing the cross-correlation of two measured time series it is necessary to replace the unbounded integration domain with a finite domain. This leads a modified definition of the cross-correlation, $f_{1}(t) \star f_{2}(t)=\int_{0}^{T} d \tau f_{1}(\tau) f_{2}(\tau+t)$. The $*$ and $\star$ notation introduced here is used below. It is often convenient to normalize $f_{1}(t) \star f_{2}(t)$ by dividing by $T$; when this is done, division by $T$ will be shown explicitly.

The starting point of our analysis is the linearized water wave equations of motion (see, e.g., [43). Let $\mathbf{x}=(x, y)$ denote lateral position vector, and assume that the vertical coordinate $z$ increases upwards, with $z=0$ at the undisturbed free surface. Let $\nabla^{2}$ denote the three dimensional Laplacian operator, and let $\nabla_{\perp}$ and $\nabla_{\perp}^{2}$ denote the two-dimensional - in $(x, y)$ - gradient and Laplacian operators, respectively. We assume that the water depth $h$ is constant. Let $\Phi(\mathbf{x}, z, t)$ denote the velocity potential and $\bar{\Phi}(\mathbf{x}, z, \omega)$ its Fourier transform. The problem is to solve $\nabla^{2} \Phi(\mathbf{x}, z, t)=f(\mathbf{x}, z, t)$ subject to $\partial^{2} \Phi / \partial t^{2}+g \partial \Phi / \partial z=0$ at $z=0$, and $\partial \Phi / \partial z=0$ at $z=-h$. The free surface displacement $\eta(\mathbf{x}, t)$ is equal to $(-1 / g) \partial \Phi / \partial t$ evaluated at $z=0 . \quad f(\mathbf{x}, z, t)$ is a source function; $\rho f$ is the rate at which mass per unit volume is injected. The problem as defined ${ }_{85}$ so far is poorly posed. The difficulty is that most functions $f(\mathbf{x}, z, t)$ don't couple naturally to small amplitude surface gravity waves, and therefore don't allow the specified free surface and bottom boundary conditions to be satisfied. Instead, most functions $f(\mathbf{x}, z, t)$ lead to a localized transient non-wave-like response. To avoid this difficulty, we shall assume that the depth dependence 90 of the source function $f(\mathbf{x}, z, t)$ is chosen to couple naturally to small amplitude surface gravity waves. (It is worth noting that the same assumption is made below in a less obvious way when we find the surface displacement Green's 
function as the solution to an initial value problem; in both cases the depth dependence of the velocity field is constrained to be dynamically consistent with the free surface displacement.)

In the frequency domain, the problem is to solve $\nabla^{2} \bar{\Phi}(\mathbf{x}, z, \omega)=\bar{f}(\mathbf{x}, z, \omega)$ subject to the boundary conditions $\omega^{2} \bar{\Phi}=g \partial \bar{\Phi} / \partial z$ at $z=0$, and $\partial \bar{\Phi} / \partial z=0$ at $z=-h$. We shall assume that $\bar{f}(\mathbf{x}, z, \omega)=\bar{p}(\mathbf{x}, \omega) \cosh k(z+h) / \cosh k h$ and that $\bar{\Phi}(\mathbf{x}, z, \omega)=\bar{\phi}(\mathbf{x}, \omega) \cosh k(z+h) / \cosh k h$. With these assumptions the boundary conditions are satisfied provided the dispersion relation $\omega^{2}=$ $g k \tanh k h$ is satisfied, and the problem is reduced to solving $\left(\nabla_{\perp}^{2}+k^{2}\right) \bar{\phi}(\mathbf{x}, \omega)=$ $\bar{p}(x, \omega)$. The solution can be written $\bar{\phi}(\mathbf{x}, \omega)=\iint d \mathbf{x}^{\prime} \bar{G}_{\phi}\left(\mathbf{x} \mid \mathbf{x}^{\prime}, \omega\right) \bar{p}\left(\mathbf{x}^{\prime}, \omega\right)$ where $\bar{G}_{\phi}\left(\mathbf{x} \mid \mathbf{x}^{\prime}, \omega\right)$ is the Green's function for the Helmholtz equation in two space dimensions,

$$
\left(\nabla_{\perp}^{2}+k^{2}\right) \bar{G}_{\phi}\left(\mathbf{x} \mid \mathbf{x}^{\prime}, \omega\right)=\delta\left(\mathbf{x}-\mathbf{x}^{\prime}\right) .
$$

In anticipation of our focus below on the free surface displacement, we introduce the free surface displacement Green's function $\bar{G}_{\eta}\left(\mathbf{x} \mid \mathbf{x}^{\prime}, \omega\right)$. Consistent with the free-surface boundary condition, $\bar{\eta}(\mathbf{x}, \omega)=(i \omega / g) \bar{\phi}(\mathbf{x}, \omega)$, we set $\bar{G}_{\eta}\left(\mathbf{x} \mid \mathbf{x}^{\prime}, \omega\right)=(i \omega / g) \bar{G}_{\phi}\left(\mathbf{x} \mid \mathbf{x}^{\prime}, \omega\right)$ and $\bar{\eta}(\mathbf{x}, \omega)=\iint d \mathbf{x}^{\prime} \bar{G}_{\eta}\left(\mathbf{x} \mid \mathbf{x}^{\prime}, \omega\right) \bar{p}\left(\mathbf{x}^{\prime}, \omega\right)$. In the time domain this corresponds to

$$
\eta(\mathbf{x}, t)=\iint d \mathbf{x}^{\prime} G_{\eta}\left(\mathbf{x} \mid \mathbf{x}^{\prime}, t\right) * p\left(\mathbf{x}^{\prime}, t\right),
$$

where $G_{\eta}\left(\mathbf{x} \mid \mathbf{x}^{\prime}, t\right)=(-1 / g)(\partial / \partial t) G_{\phi}\left(\mathbf{x} \mid \mathbf{x}^{\prime}, t\right)$. The preceding discussion serves to define the relevant Green's functions and their relationship to measurable quantities.

\subsection{General derivation of Eq. (1)}

We now present a derivation of Eq. (1) that holds in both open and closed systems. We begin with a derivation of the Green's function identity, Eq. (12), that underlies Eq. (1). In the presence of weak dissipation, which is assumed to be proportional to $\omega$ times the parameter $\epsilon$, the Green's function $\bar{G}_{\phi}\left(\mathbf{x} \mid \mathbf{x}_{0}, \omega\right)$ at $\mathbf{x}$, corresponding to point source excitation at $\mathbf{x}_{0}$, satisfies 
$\left(\nabla_{\perp}^{2}+k^{2}+i \omega \epsilon\right) \bar{G}_{\phi}\left(\mathbf{x} \mid \mathbf{x}_{0}, \omega\right)=\delta\left(\mathbf{x}-\mathbf{x}_{0}\right)$. Thus, $\bar{G}_{\phi}$ at point $\mathbf{x}$ excited by a point source at $\mathbf{x}_{A}$ satisfies

$$
\left(\nabla_{\perp}^{2}+k^{2}+i \omega \epsilon\right) \bar{G}_{\phi}\left(\mathbf{x} \mid \mathbf{x}_{A}, \omega\right)=\delta\left(\mathbf{x}-\mathbf{x}_{A}\right)
$$

Similarly, $\bar{G}_{\phi}$ at point $\mathbf{x}$ excited by a point source at $\mathbf{x}_{B}$ satisfies

$$
\left(\nabla_{\perp}^{2}+k^{2}+i \omega \epsilon\right) \bar{G}_{\phi}\left(\mathbf{x} \mid \mathbf{x}_{B}, \omega\right)=\delta\left(\mathbf{x}-\mathbf{x}_{B}\right) .
$$

Due to reciprocity $\bar{G}_{\phi}\left(\mathbf{x} \mid \mathbf{x}_{A}, \omega\right)$ is equal to $\bar{G}_{\phi}\left(\mathbf{x}_{A} \mid \mathbf{x}, \omega\right)$, and $\bar{G}_{\phi}\left(\mathbf{x} \mid \mathbf{x}_{B}, \omega\right)$ is equal to $\bar{G}_{\phi}\left(\mathbf{x}_{B} \mid \mathbf{x}, \omega\right)$. Multiplication of $(4)$ by $\bar{G}_{\phi}^{*}\left(\mathbf{x}_{B} \mid \mathbf{x}, \omega\right)$, followed by integration over $\mathbf{x}$, and complex conjugation of left- and right-hand sides gives

$$
\iint d \mathbf{x} \bar{G}_{\phi}\left(\mathbf{x}_{B} \mid \mathbf{x}, \omega\right)\left(\nabla_{\perp}^{2}+k^{2}-i \omega \epsilon\right) \bar{G}_{\phi}^{*}\left(\mathbf{x}_{A} \mid \mathbf{x}, \omega\right)=\bar{G}_{\phi}\left(\mathbf{x}_{B} \mid \mathbf{x}_{A}, \omega\right) .
$$

125

Multiplication of $(5)$ by $\bar{G}_{\phi}^{*}\left(\mathbf{x}_{A} \mid \mathbf{x}, \omega\right)$, followed by integration over $\mathbf{x}$ gives

$$
\iint d \mathbf{x} \bar{G}_{\phi}^{*}\left(\mathbf{x}_{A} \mid \mathbf{x}, \omega\right)\left(\nabla_{\perp}^{2}+k^{2}+i \omega \epsilon\right) \bar{G}_{\phi}\left(\mathbf{x}_{B} \mid \mathbf{x}, \omega\right)=\bar{G}_{\phi}^{*}\left(\mathbf{x}_{A} \mid \mathbf{x}_{B}, \omega\right) .
$$

Subtracting (7) from (6) gives

$$
Q-2 i \omega \epsilon \iint d \mathbf{x} \bar{G}_{\phi}\left(\mathbf{x}_{B} \mid \mathbf{x}, \omega\right) \bar{G}_{\phi}^{*}\left(\mathbf{x}_{A} \mid \mathbf{x}, \omega\right)=\bar{G}_{\phi}\left(\mathbf{x}_{B} \mid \mathbf{x}_{A}, \omega\right)-\bar{G}_{\phi}^{*}\left(\mathbf{x}_{A} \mid \mathbf{x}_{B}, \omega\right) .
$$

where

$$
\begin{aligned}
Q & =\iint d \mathbf{x} \bar{G}_{\phi}\left(\mathbf{x}_{B} \mid \mathbf{x}, \omega\right) \nabla_{\perp}^{2} \bar{G}_{\phi}^{*}\left(\mathbf{x}_{A} \mid \mathbf{x}, \omega\right)-\iint d \mathbf{x} \bar{G}_{\phi}^{*}\left(\mathbf{x}_{A} \mid \mathbf{x}, \omega\right) \nabla_{\perp}^{2} \bar{G}_{\phi}\left(\mathbf{x}_{B} \mid \mathbf{x}, \omega\right) \\
& =\oint d l\left[\bar{G}_{\phi}\left(\mathbf{x}_{B} \mid \mathbf{x}, \omega\right) \nabla_{\perp} \bar{G}_{\phi}^{*}\left(\mathbf{x}_{A} \mid \mathbf{x}, \omega\right)-\bar{G}_{\phi}^{*}\left(\mathbf{x}_{A} \mid \mathbf{x}, \omega\right) \nabla_{\perp} \bar{G}_{\phi}\left(\mathbf{x}_{B} \mid \mathbf{x}, \omega\right)\right] \cdot \hat{n}
\end{aligned}
$$

By reciprocity $\bar{G}_{\phi}\left(\mathbf{x}_{B} \mid \mathbf{x}_{A}, \omega\right)=\bar{G}_{\phi}\left(\mathbf{x}_{A} \mid \mathbf{x}_{B}, \omega\right)$, so the rhs of $(8)$ can be written $2 \operatorname{Im} \bar{G}_{\phi}\left(\mathbf{x}_{B} \mid \mathbf{x}_{A}, \omega\right)$. The second form of $Q$ given above follows from applying the divergence theorem to $\iint d \mathbf{x} \nabla_{\perp} \cdot\left[\bar{G}_{\phi}\left(\mathbf{x}_{B} \mid \mathbf{x}, \omega\right) \nabla_{\perp} \bar{G}_{\phi}^{*}\left(\mathbf{x}_{A} \mid \mathbf{x}, \omega\right)-\right.$ $\left.\bar{G}_{\phi}^{*}\left(\mathbf{x}_{A} \mid \mathbf{x}, \omega\right) \nabla_{\perp} \bar{G}_{\phi}\left(\mathbf{x}_{B} \mid \mathbf{x}, \omega\right)\right]$. The integral in the latter form of $Q$ is a line integral over the boundary of the $\mathbf{x}$-domain and $\hat{n}$ is a unit outward normal. We consider two choices for the domain of $\mathbf{x}$. First, appropriate for open ocean conditions, consider the domain enclosed within a large circle of radius $R$ cen- 
large $R, \bar{G}_{\phi}$ and $\nabla_{\perp} \bar{G}_{\phi} \cdot \hat{n}$ approach zero faster than $R^{-1 / 2}$, so $Q$ approaches 0 . Second, consider an $\mathbf{x}$-domain bounded by rigid walls that enclose $\mathbf{x}_{A}$ and $\mathbf{x}_{B}$, as in a wavetank. Because $\nabla_{\perp} \bar{G}_{\phi} \cdot \hat{n}=0$ at rigid walls, each term in $Q$ vanishes so $Q=0$. Setting $Q=0$ in (8) gives

$$
-2 i \omega \epsilon \iint d \mathbf{x} \bar{G}_{\phi}\left(\mathbf{x}_{B} \mid \mathbf{x}, \omega\right) \bar{G}_{\phi}^{*}\left(\mathbf{x}_{A} \mid \mathbf{x}, \omega\right)=\bar{G}_{\phi}\left(\mathbf{x}_{B} \mid \mathbf{x}_{A}, \omega\right)-\bar{G}_{\phi}^{*}\left(\mathbf{x}_{A} \mid \mathbf{x}_{B}, \omega\right) .
$$

Transforming to the time domain gives

$$
2 \epsilon \frac{d}{d t} \iint d \mathbf{x} G_{\phi}\left(\mathbf{x}_{B} \mid \mathbf{x}, t\right) * G_{\phi}\left(\mathbf{x}_{A} \mid \mathbf{x},-t\right)=G_{\phi}\left(\mathbf{x}_{B} \mid \mathbf{x}_{A}, t\right)-G_{\phi}\left(\mathbf{x}_{B} \mid \mathbf{x}_{A},-t\right) .
$$

where $*$ (not a superscript) denotes convolution. Recall that the convolution of $G_{\phi}\left(\mathbf{x}_{B} \mid \mathbf{x}, t\right)$ and $G_{\phi}\left(\mathbf{x}_{A} \mid \mathbf{x},-t\right)$ is equivalent to the cross-correlation of $G_{\phi}\left(\mathbf{x}_{B} \mid \mathbf{x}, t\right)$ and $G_{\phi}\left(\mathbf{x}_{A} \mid \mathbf{x}, t\right)$.

The above considerations focus on the Green's function for the velocity potential evaluated at the free surface, $G_{\phi}$. From a measurement point of view it is more natural to focus on the surface elevation Green's function, $G_{\eta}$. Recall that $\bar{\eta}(\mathbf{x})=(i \omega / g) \bar{\phi}(\mathbf{x}), \bar{G}_{\eta}=(i \omega / g) \bar{G}_{\phi}$, and $G_{\eta}=(-1 / g) \partial G_{\phi} / \partial t$. Using these relationships, Eqs. (9) and (10) are equivalent to

$$
2 \epsilon g \iint d \mathbf{x} \bar{G}_{\eta}\left(\mathbf{x}_{B} \mid \mathbf{x}, \omega\right) \bar{G}_{\eta}^{*}\left(\mathbf{x}_{A} \mid \mathbf{x}, \omega\right)=\bar{G}_{\eta}\left(\mathbf{x}_{B} \mid \mathbf{x}_{A}, \omega\right)+\bar{G}_{\eta}^{*}\left(\mathbf{x}_{A} \mid \mathbf{x}_{B}, \omega\right)
$$

and

$$
2 \epsilon g \iint d \mathbf{x} G_{\eta}\left(\mathbf{x}_{B} \mid \mathbf{x}, t\right) * G_{\eta}\left(\mathbf{x}_{A} \mid \mathbf{x},-t\right)=G_{\eta}\left(\mathbf{x}_{B} \mid \mathbf{x}_{A}, t\right)+G_{\eta}\left(\mathbf{x}_{B} \mid \mathbf{x}_{A},-t\right),
$$

respectively. Equation 12 is the Green's function identity that we set out to prove.

The dissipation $\epsilon$ plays a subtle role in Eqs. 9 - 12). On an unbounded domain the integrals over $\mathbf{x}$ in those equations diverge if $\epsilon=0$, so the introduction of a small but nonzero dissipation prevents the occurrence of indeterminate products (zero times infinity). For numerical purposes, e.g., to test 12. one might evaluate $2 g \iint d \mathbf{x} G_{\eta}\left(\mathbf{x}_{B} \mid \mathbf{x}, t\right) * G_{\eta}\left(\mathbf{x}_{A} \mid \mathbf{x},-t\right)$ using the large circular domain described above and compute the Green's functions assuming 
no dissipation. To a very good approximation the result will be $P(R)$ times $G_{\eta}\left(\mathbf{x}_{B} \mid \mathbf{x}_{A}, t\right)+G_{\eta}\left(\mathbf{x}_{B} \mid \mathbf{x}_{A},-t\right)$, where $P(R)$ grows with increasing domain radius $R$ and has the dimensions of $\epsilon^{-1}$, or $\mathrm{m}^{2} / \mathrm{s}$. For the purposes described in this paper, the relevant spatial domains and integration times are finite, and proportionality of the left- and right-hand sides of 12 is all that is required. With these comments in mind, for our purposes there is no need to account for dissipation when computing the relevant Green's functions.

In an unbounded domain and in the limit $\epsilon=0, \bar{G}_{\phi}\left(\mathbf{x} \mid \mathbf{x}_{0}, \omega\right)=(-i / 4) H_{0}^{(1)}(k r)$ where $r=\left|\mathbf{x}-\mathbf{x}_{0}\right|$ and $H_{0}^{(1)}$ is the Hankel function of the first kind. Thus, $\left.G_{\phi}\left(\mathbf{x} \mid \mathbf{x}_{0}, t\right)=F^{-1}\left[(-i / 4) c_{g}^{-1}(k(\omega))\right] H_{0}^{(1)}(k(\omega) r)\right]$. (The factor $c_{g}^{-1}(k(\omega))=$ $(\partial \omega / \partial k)^{-1}$ is the Jacobian of the the transformation from $k$ to $\omega$. Also, when evaluating the Fourier integral, the constraint on negative frequencies noted above insures that $G_{\phi}\left(\mathbf{x} \mid \mathbf{x}_{0}, t\right)$ is real). $G_{\phi}\left(\mathbf{x} \mid \mathbf{x}_{0}, t\right)$ constructed in this way is well-defined provided $k(\omega)$ is single-valued. A caveat is discussed below.

We now complete the derivation of the correlation function result (1). Equations (11) and $(12$ are mathematical identities. In contrast, the corresponding correlation function result is an approximate result whose validity depends on Eq. 12 and additional statistical assumptions, as described below. It follows from Eq. (3) that the surface displacement at $\mathbf{x}_{A}$ can be written $\eta\left(\mathbf{x}_{A}, t\right)=\iint d \mathbf{x} G_{\eta}\left(\mathbf{x}_{A} \mid \mathbf{x}, t\right) * p(\mathbf{x}, t)$ where $p(\mathbf{x}, t)$ is the source function. Similarly, $\eta\left(\mathbf{x}_{B}, t\right)=\iint d \mathbf{x} G_{\eta}\left(\mathbf{x}_{B} \mid \mathbf{x}, t\right) * p(\mathbf{x}, t)$. Let $C_{A B}(t)$ denote the crosscorrelation of $\eta\left(\mathbf{x}_{A}, t\right)$ and $\eta\left(\mathbf{x}_{B}, t\right)$. Then

$$
C_{A B}(t)=\iint d \mathbf{x} \iint d \mathbf{x}^{\prime} G_{\eta}\left(\mathbf{x}_{A} \mid \mathbf{x}, t\right) * G_{\eta}\left(\mathbf{x}_{B} \mid \mathbf{x}^{\prime},-t\right) * p(\mathbf{x}, t) * p\left(\mathbf{x}^{\prime},-t\right) .
$$

We shall assume that the source function is delta-correlated in space and nearly so in time,

$$
p(\mathbf{x}, t) * p\left(\mathbf{x}^{\prime},-t\right)=a^{2} \delta\left(\mathbf{x}-\mathbf{x}^{\prime}\right) D(t),
$$

where $D(t)$ is a band-limited approximation to a delta function. If the assumed random sources have time history $d(t)$, then $D(t)=d(t) * d(-t)$. Note that $\bar{D}(\omega)=\bar{d}(\omega) \bar{d}^{*}(\omega)=|\bar{d}(\omega)|^{2}$, so any phase information contained in $d(t)$ is lost in $D(t)$. The quantity $a$ has dimension m. Both $d(t)$ and $D(t)$ have dimension 
$\mathrm{s}^{-1}$; in the limiting case of infinite bandwidth, $D(t) \rightarrow \delta(t)$. Making use of Eq. (14) in Eq. 13), the latter equation reduces to

$$
C_{A B}(t)=a^{2} D(t) * \iint d \mathbf{x} G_{\eta}\left(\mathbf{x}_{A} \mid \mathbf{x}, t\right) * G_{\eta}\left(\mathbf{x}_{B} \mid \mathbf{x},-t\right) .
$$

Comparison of Eqs. 12 and 15 then yields

$$
\frac{2 \epsilon g}{a^{2}} C_{A B}(t)=D(t) *\left[G_{\eta}\left(\mathbf{x}_{B} \mid \mathbf{x}_{A}, t\right)+G_{\eta}\left(\mathbf{x}_{B} \mid \mathbf{x}_{A},-t\right)\right]
$$

which is seen to be identical to Eq. (1) for a particular choice of the constant $\gamma$.

In practice, the correlation function $C_{A B}(t)$ is evaluated as $\eta\left(\mathbf{x}_{A}, t\right) \star \eta\left(\mathbf{x}_{B}, t\right)$. The comments made above about expecting only proportionality of the lhs and rhs of Eq. 12 apply also to Eq. (16). Note that Eq. 12) is exact (within a linear theory framework), while the validity of Eq. (16) rests on the idealized statistical assumption, Eq. (14), that we have made about the source function $v(\mathbf{x}, t)$. Experiments with other wave types suggest that Eq. 16 should be a good approximation even when those assumptions are relaxed.

\subsection{Alternate derivation of Eq. (1) for a closed system}

We consider now an alternative derivation of Eq. (1), appropriate for a bounded domain, such as the wavetank geometry described above. Although this alternate derivation is less general than the one presented above, it is useful for two reasons. First, it gives additional insight into Eq. (1). Second, some of the results presented in this subsection are used as the basis for numerical results presented in the two sections that follow. Unlike the derivation presented above, the following derivation (see also [2] requires that we first explicitly solve for the Green's function. In a rectangular basin, $0 \leq x \leq L_{x}$ and $0 \leq y \leq L_{y}$, whose depth is $h$, the velocity potential

$\Phi(x, y, z, t)=g \sum_{l=0}^{\infty} \sum_{m=0}^{\infty} \frac{\cosh k_{l m}(z+h)}{\cosh k_{l m} h} \cos \left(\frac{l \pi x}{L_{x}}\right) \cos \left(\frac{m \pi y}{L_{y}}\right)\left(a_{l m} \cos \omega_{l m} t+b_{l m} \sin \omega_{l m} t\right)$

satisfies the relevant lateral boundary conditions and $\nabla^{2} \Phi=0$ provided $\left(l \pi / L_{x}\right)^{2}+$ $\left(m \pi / L_{y}\right)^{2}=k_{l m}^{2}$. The linearized free surface boundary condition is satisfied provided $\omega_{l m}^{2}=g k_{l m} \tanh k_{l m} h$. The vertical component of fluid velocity $\partial \Phi / \partial z$ 


$$
\eta(x, y, t)=\sum_{l=0}^{\infty} \sum_{m=0}^{\infty} \omega_{l m} \cos \left(\frac{l \pi x}{L_{x}}\right) \cos \left(\frac{m \pi y}{L_{y}}\right)\left(a_{l m} \sin \omega_{l m} t-b_{l m} \cos \omega_{l m} t\right) .
$$

The $l=m=0$ term can be neglected in 17 because $\omega_{00}=0$. It follows from Eq. (3) that $G_{\eta}\left(\mathbf{x} \mid \mathbf{x}_{0}, t\right)$ is $1 / A_{0}$ times the particular choice of $\eta(\mathbf{x}, t)$ for which the source function $p(\mathbf{x}, t)=A_{0} \delta\left(\mathbf{x}-\mathbf{x}_{0}\right) \delta(t)$. ( $A_{0}$ has dimension $\mathrm{m}^{2}$, but dependence on $A_{0}$ cancels in the resulting expression for $G_{\eta}(\mathbf{x}, t)$.) Physically, this source function corresponds to an instantaneous injection of a volume $V_{0}=L_{0} A_{0}$ of water at $\mathbf{x}=\mathbf{x}_{0}, t=0$. The Green's function $G_{\eta}\left(\mathbf{x} \mid \mathbf{x}_{0}, t\right)$ can be constructed from Eq. (17) as $1 / A_{0}$ times the solution, $\eta(\mathbf{x}, t)$, of the initial value problem, $\eta(\mathbf{x}, 0)=V_{0} \delta\left(\mathbf{x}-\mathbf{x}_{0}\right)$ and $(\partial \eta(\mathbf{x}, t) / \partial t)(\mathbf{x}, 0)=0$. The constants $a_{l m}$ and $b_{l m}$, whose units are $\mathrm{m} \times \mathrm{s}$, are found by making use of the orthogonality of the modes. After some algebra one finds

$G_{\eta}\left(\mathbf{x} \mid \mathbf{x}^{\prime}, t\right)=\frac{L_{0}}{L_{x} L_{y}} H(t) \sum_{l} \sum_{m} \beta_{l} \beta_{m} \cos \left(\frac{l \pi x}{L_{x}}\right) \cos \left(\frac{m \pi y}{L_{y}}\right) \cos \left(\frac{l \pi x^{\prime}}{L_{x}}\right) \cos \left(\frac{m \pi y^{\prime}}{L_{y}}\right) \cos \omega_{l m} t$

where $\beta_{l}=1$ for $l=0, \beta_{l}=2$ for $l=1,2, \ldots$, and $H(t)$ is the Heaviside step function. Equation (18) is a special case of a more general modal representation of the Green's function,

$$
G_{\eta}\left(\mathbf{x} \mid \mathbf{x}^{\prime}, t\right)=\frac{L_{0}}{A} H(t) \sum_{n} \psi_{n}(\mathbf{x}) \psi_{n}\left(\mathbf{x}^{\prime}\right) \cos \omega_{n} t
$$

where $A$ is the lateral area of the domain. The dimensionless orthogonal modes 225 satisfy the relevant boundary conditions and are assumed to be normalized, $\iint d \mathbf{x} \psi_{n}(\mathbf{x}) \psi_{m}(\mathbf{x})=A \delta_{n m}$. In the following we shall make use of the more general and more convenient expression (19), noting that Eq. (18) is a special case.

We now demonstrate that the Green's function representation $[19$ is consistent with the Green's function identity 12 . After some reordering of terms and making use of the modal orthogonality condition, it follows from Eq. (19) 
that

$\iint d \mathbf{x} G_{\eta}\left(\mathbf{x}_{A} \mid \mathbf{x}, t\right) \star G_{\eta}\left(\mathbf{x}_{B} \mid \mathbf{x}, t\right)=\frac{L_{0}}{A} \sum_{n} \psi_{n}\left(\mathbf{x}_{A}\right) \psi_{n}\left(\mathbf{x}_{B}\right)\left[H(t) \cos \omega_{n} t \star H(t) \cos \omega_{n} t\right]$.

Evaluation of the cross-correlation $H(t) \cos \omega_{n} t \star H(t) \cos \omega_{n} t$ requires some care due to the one-sided nature of the functions involved:

$$
\begin{aligned}
H(t) \cos \omega_{n} t \star H(t) \cos \omega_{n} t & =\int_{0}^{T} d \tau \cos \omega_{n} \tau\left[\cos \omega_{n}(\tau+t)+\cos \omega_{n}(\tau-t)\right] \\
& =2 \cos \omega_{n} t \int_{0}^{T} d \tau \cos ^{2} \omega_{n} \tau \\
& =T \cos \omega_{n} t\left(1+\frac{\sin \omega_{n} T \cos \omega_{n} T}{\omega_{n} T}\right)
\end{aligned}
$$

235 We shall assume that $\omega_{n} T$ is large so the second term in square brackets in 21) can be neglected. Noting the one-sided nature of the Green's function 19, substituting (21) into (20) gives

$$
\frac{L_{0}}{T} \iint d \mathbf{x} G_{\eta}\left(\mathbf{x}_{A} \mid \mathbf{x}, t\right) \star G_{\eta}\left(\mathbf{x}_{B} \mid \mathbf{x}, t\right)=G_{\eta}\left(\mathbf{x}_{A} \mid \mathbf{x}_{B}, t\right)+G_{\eta}\left(\mathbf{x}_{A} \mid \mathbf{x}_{B},-t\right) .
$$

Apart from an unimportant constant, Eq. 22 is seen to be identical to Eq. 12. Recall that the cross-correlation $G_{\eta}\left(\mathbf{x}_{A} \mid \mathbf{x}, t\right) \star G_{\eta}\left(\mathbf{x}_{B} \mid \mathbf{x}, t\right)$ is an integral over a finite time interval $T$. Had we considered the limit $T \rightarrow \infty$, we would have encountered an indeterminate form (zero times infinity) on the left hand side of Eq. [22), as discussed earlier.

The argument involving Eqs. (13) and (15) that leads to Eq. (16) is independent of the form of $G_{\eta}\left(\mathbf{x} \mid \mathbf{x}^{\prime}, t\right)$, so that argument applies when the Eq. (19) 245 representation of $G_{\eta}\left(\mathbf{x} \mid \mathbf{x}^{\prime}, t\right)$ is valid. To supplement that argument, we now provide an alternate derivation of the correlation result, Eq. (16), that makes use of 19). A random wavefield can be described as a superposition of modes with random phases,

$$
\eta(\mathbf{x}, t)=d(t) * \sum_{n}\left(c_{n} \cos \omega_{n} t+d_{n} \sin \omega_{n} t\right) \psi_{n}(\mathbf{x}) .
$$

The random phase assumption dictates that

$$
\left\langle c_{n} c_{m}\right\rangle=\left\langle d_{n} d_{m}\right\rangle=a^{2} \delta_{n m},\left\langle c_{n} d_{m}\right\rangle=0
$$




\section{Simulations}

In this section we present simulations to test the validity of Eq. (1) in both open and closed systems. First we consider an open system, consisting of a laterally unbounded domain. We consider both deep and nearly-shallow 270

where \langle\rangle denotes ensemble average. Convolution with $d(t)$ is included in Eq. (23) to account for the fact that any physically realizable wave field must have finite bandwidth. As was the case following Eq. (14), we assume that $d(t)$ has dimension $\mathrm{s}^{-1}$. Then, because the normalized modes $\psi_{n}(\mathbf{x})$ are dimensionless, $c_{n}, d_{n}$ and $a$ have dimension $m$. Although specification of $d(t)$ includes phase information, that information is lost in $\eta(\mathbf{x}, t)$ because $c_{n}$ and $d_{n}$ have random phases. This observation is consistent with Eqs. 26) and 27 below, because, as noted in the discussion following Eq. (14), $D(t)$ does not depend on the phase information contained in $d(t)$. It is natural to define the cross-correlation of measurements of surface displacement at locations $\mathbf{x}_{A}$ and $\mathbf{x}_{B}$ as an ensemble average,

$$
C_{A B}(t)=\left\langle\int_{0}^{T} d \tau \eta\left(\mathbf{x}_{A}, \tau\right) \eta\left(\mathbf{x}_{B}, \tau+t\right)\right\rangle .
$$

Substituting Eq. 23 into Eq. 25 and making use of the assumed statistical properties of the $c_{n}$ 's and $d_{n}$ 's, Eq. (24), gives, after some algebra,

$$
C_{A B}(t)=D(t) * a^{2} T \sum_{n} \psi_{n}\left(\mathbf{x}_{A}\right) \psi_{n}\left(\mathbf{x}_{B}\right) \cos \omega_{n} t
$$

where $D(t)=d(t) * d(-t)$. Comparison of this expression with Eq. 19 gives

$$
\frac{L_{0}}{A a^{2} T} C_{A B}(t)=D(t) *\left[G_{\eta}\left(\mathbf{x}_{B} \mid \mathbf{x}_{A}, t\right)+G_{\eta}\left(\mathbf{x}_{B} \mid \mathbf{x}_{A},-t\right)\right]
$$

Like Eq. (16), Eq. (27) is seen to be identical to Eq. (1) for a particular choice of the constant $\gamma$.

conditions. Recall that $\bar{G}_{\eta}=(i \omega / g) \bar{G}_{\phi}$, and that in an unbounded domain $\bar{G}_{\phi}\left(\mathbf{x} \mid \mathbf{x}_{0}, \omega\right)=(-i / 4) H_{0}^{(1)}(k(\omega) r)$ where $r=\left|\mathbf{x}-\mathbf{x}_{0}\right|$. It follows that

$$
G_{\eta}\left(\mathbf{x} \mid \mathbf{x}_{0}, t\right)=\frac{1}{4 g} F^{-1}\left[\frac{\omega}{c_{g}(k(\omega))} H_{0}^{(1)}(k(\omega) r)\right] .
$$


More generally, we wish to compute the response, $d(t) * G_{\eta}\left(\mathbf{x} \mid \mathbf{x}_{0}, t\right)$, to a bandlimited point source. For numerical purposes, we set (neglecting an unimportant dimensional constant) $\bar{d}(\omega)=\left(\sin \left(0.5 \omega / f_{\max }\right)\right)^{1 / 2}$ with wave frequencies, $f=$ $275 \omega / 2 \pi$, limited to the band $0<f<f_{\max }$. The convolution $d(t) * G_{\eta}\left(\mathbf{x} \mid \mathbf{x}_{0}, t\right)$ has the general form $F^{-1}[\bar{b}(\omega)]$; to insure that $d(t) * G_{\eta}\left(\mathbf{x} \mid \mathbf{x}_{0}, t\right)$ is real, the condition $\bar{b}(-\omega)=\bar{b}^{*}(\omega)$ is applied in our numerical simulations.

To test Eq. (1) a circular domain with $1500 \mathrm{~m}$ radius, centered at $x=$ $y=0$, was used. The field points of interest were assumed to be at $\mathbf{x}_{A}=$ $(-25 \mathrm{~m}, 0)$ and $\mathbf{x}_{B}=(25 \mathrm{~m}, 0)$. A random field of waves was constructed by simulating the response to approximately $\pi / 4 \times 10^{6}$ quasi-impulsive point sources, at positions $\mathbf{x}_{j}$ and times $t_{j}$, that were randomly distributed, with a uniform distribution, throughout the circular domain and throughout a 409600 s (about 114 hours) time window. $\eta\left(\mathbf{x}_{A}, t\right)$ was computed as the sum (over $j$ ) of contributions, $d(t) * G_{\eta}\left(\mathbf{x}_{A} \mid \mathbf{x}_{j}, t-t_{j}\right)$, and similarly for $\eta\left(\mathbf{x}_{B}, t\right) . \eta\left(\mathbf{x}_{A}, t\right)$ and $\eta\left(\mathbf{x}_{B}, t\right)$ computed in this fashion look like band-limited noise (as expected). In two different environments the cross-correlation $C_{A B}(t)$ of $\eta\left(\mathbf{x}_{A}, t\right)$ and $\eta\left(\mathbf{x}_{B}, t\right)$ was computed by coherently averaging approximately 5000 realizations of the correlation function, each based on $160 \mathrm{~s}$ blocks of simulated surface elevation at ${ }_{290} \mathbf{x}_{A}$ and $\mathbf{x}_{B}$; the resulting $C_{A B}(t)$ estimates are shown in Figs. 1 and 2. In those figures $C_{A B}(t)$ is compared to $D(t) *\left[G_{\eta}\left(\mathbf{x}_{B} \mid \mathbf{x}_{A}, t\right)+G_{\eta}\left(\mathbf{x}_{A} \mid \mathbf{x}_{B},-t\right)\right]$, where $D(t)=d(t) * d(-t)$. Like the random wave field just described, the Green's function $G_{\eta}\left(\mathbf{x}_{B} \mid \mathbf{x}_{A}, t\right)=G_{\eta}\left(\mathbf{x}_{A} \mid \mathbf{x}_{B}, t\right)$ was constructed using Eq. 28). Figure 1 corresponds to a water depth of $1.0 \mathrm{~m}$ with $f_{\max }=0.3 \mathrm{~Hz}$. To account for weak dispersion in this frequency band, the approximate dispersion relation, $\omega^{2}=g h k^{2}\left(1-(k h)^{2} / 3\right)$, was used. $C_{A B}(t)$ in Fig. 1 is seen to be in very good agreement with $D(t) *\left[G_{\eta}\left(\mathbf{x}_{B} \mid \mathbf{x}_{A}, t\right)+G_{\eta}\left(\mathbf{x}_{A} \mid \mathbf{x}_{B},-t\right)\right]$, as predicted by Eq. (16) or (1). Figure 2 corresponds to deep water conditions, $\omega^{2}=g k$, with $f_{\max }=0.5 \mathrm{~Hz}$. Again, agreement between $C_{A B}(t)$ and $D(t) *\left[G_{\eta}\left(\mathbf{x}_{B} \mid \mathbf{x}_{A}, t\right)+\right.$ $\left.{ }_{300} G_{\eta}\left(\mathbf{x}_{A} \mid \mathbf{x}_{B},-t\right)\right]$ is seen to be very good. A caveat is discussed below. Note that, owing to dispersion, the deep water Green's function shown in Fig. 2 consists of a simple frequency-modulated sweep with frequency increasing with increasing 
time.

We consider now a closed system consisting of a model of a narrow rectan-

of the calculation of $G_{\eta}\left(x_{B} \mid x_{A}, t\right)$. The procedure just described was followed using $L_{x}=11.9 \mathrm{~m}, L_{y}=1 \mathrm{~m}, h=0.42 \mathrm{~m}, x_{A}=2.38 \mathrm{~m}$ and $x_{B}=9.52$ $\mathrm{m}$. The random variables $c_{l}$ and $d_{l}$ were Gaussian random variables with zero mean and unit variance; an independent $\left(c_{l}, d_{l}\right)$ pair was used in each of 240 and $\omega^{2} \exp \left(-(\omega / 3 \pi)^{2}\right)$ for $\omega \geq 3 \pi$. Results are shown in Fig. 3. In that figure only positive time lags are shown; the negative lag structure is essentially identical to the positive lag structure shown. Agreement between the average 
3зо $C_{A B}(t)$ and $D(t) * G\left(x_{B} \mid x_{A}, t\right)$ is seen to be excellent, as predicted by Eq. 27 or 11.

\section{Wave tank experiment}

An experiment was performed in a wave tank to test Eq. (1). In the experiment, the parameters $L_{x}, L_{y}, h, x_{A}, x_{B}$ were identical to those used in the simulations just described. The calculation of $G_{\eta}\left(x_{A} \mid x_{B}, t\right)$ is thus identical to that just described, based on Eq. 29. The effective source function $d(t)$ for the wave tank experiment was different from that used in the simulations, however, as described below.

In the experiment, a programmable wave paddle at one end $(x=0)$ of the wave tank was driven by 88 repetitions of a pseudo-random signal $s_{i}(t)$. The pseudo-random signal had the form $s_{i}(t)=F^{-1}\left[\bar{s}_{i}(\omega)\right]$, where $\bar{s}_{i}(\omega)$ was equal to $3 \pi \omega \exp (-\omega / 3 \pi) \exp \left(i \vartheta_{i}\right)$ for $\omega<3 \pi$, and $\omega^{2} \exp \left(-(\omega / 3 \pi)^{2}\right) \exp \left(i \vartheta_{i}\right)$ for $\omega \geq 3 \pi$. The phase $\vartheta_{i}$ was a random variable, uniformly distributed between 0 and $2 \pi$. In each of the 88 repetitions of $s_{i}(t)$, an independent $\vartheta_{i}$ was used. 345 Each realization of $s_{i}(t)$ had a duration of $204.8 \mathrm{~s}$. Note that $\left|\bar{s}_{i}(\omega)\right|^{2}$ is equal to $|\bar{d}(\omega)|^{2}$ that was used in the closed system simulations described in the previous section, but, unlike $d(t), s_{i}(t)$ includes a random phase. That was done to generate a random wave field in the wave tank; in the simulations, the random variables $c_{l}$ and $d_{l}$ insured that phases were random. Time histories of the surface elevation, $\eta\left(x_{A}, t\right)$ and $\eta\left(x_{B}, t\right)$, sampled at $547 \mathrm{~Hz}$, were measured using a laser-based system over the 5-hour duration of the experiment. For analysis purposes, the raw data were resampled at $50 \mathrm{~Hz}$. The data were block-processed in overlapping 204.8 s windows, with $50 \%$ overlap. Each 204.8 s block of data was treated as an independent realization of the random wave field; coherent averaging of estimates of $C_{A B}(t)$ over all realizations of the wave field was performed, as in the simulations described above. The measured spectra $\bar{\eta}\left(x_{A}, \omega\right)$ and $\bar{\eta}\left(x_{B}, \omega\right)$ were nearly identical (as predicted by Eq. 30 because in our experiment $L_{x}-x_{B}=x_{A}$ ), but those spectra were slightly different than the 
corresponding spectra in the simulations just described. This difference can be attributed to the input-voltage-to-surface-displacement transfer function. Because that transfer function is poorly known, we used an empirical approach to estimate $D(t)$ in Eq. (1) or 27). $\bar{D}(\omega)=\bar{d}(\omega) \bar{d}^{*}(\omega)$ is independent of the phase information in $\bar{d}(\omega)$, so we may, without loss of generality, assume that $\bar{d}(\omega)$ is real. Using a simple curve-fitting procedure, $\bar{d}(\omega)$ was chosen so that the theoretical spectrum $\sum_{l} \bar{d}\left(\omega_{l}\right)\left|\cos \left(l \pi x_{A} / L_{x}\right)\right|$ matches the measured average spectrum $\left(\left|\bar{\eta}\left(x_{A}, \omega\right)\right|+\left|\bar{\eta}\left(x_{B}, \omega\right)\right|\right) / 2$; the resulting fit is shown in Fig. 4. With this $\bar{d}(\omega), D(t) *\left[G_{\eta}\left(x_{B} \mid x_{A}, t\right)+G_{\eta}\left(x_{A} \mid x_{B},-t\right)\right]$ was computed and compared to the measurement-based estimate of the average $C_{A B}(t)$. To slightly improve signal-to-noise, $C_{A B}(t)$ and $C_{A B}(-t)$ were averaged and compared to

$370 D(t) * G_{\eta}\left(x_{B} \mid x_{A}, t\right)$ in the figure. The result is shown in Fig. 5; agreement is seen to be good, consistent with Eq. 27) or (1).

\section{Ocean measurements}

A natural extension of the analysis of simulations and wave tank measurements described in the previous two sections is the analysis of ocean waves. The minimum requirement that a candidate data set must satisfy is that the data must include two or more sets of concurrent wave measurements made in proximity to each other. Two data sets that satisfied this criterion were identified and analyzed. Both resulted in very disappointing results. Only one of those analysis efforts is described here. (The data set not described here was inferior in many respects to the one whose analysis is described here.)

The data set analyzed here was collected as part of the Shallow Water 2006 (SW06) experiment 44], which was a large multidisciplinary oceanographic field project conducted on the New Jersey continental shelf between July and September 2006. The surface wave data analyzed here was collected on two moored spar buoys [45] at locations $\left(39.0739^{\circ} \mathrm{N}, 73.1641^{\circ} \mathrm{W}\right)$ and $\left(39.0192^{\circ} \mathrm{N}, 73.0536^{\circ} \mathrm{W}\right)$. These were named 'Romeo' and 'Yankee', respectively. The ocean depth at both measurement locations was $80 \mathrm{~m}$, and the separation between measure- 
ment buoys was approximately $11 \mathrm{~km}$. Although approximately 10 days of data was collected, beginning on 2 August, only one 11 hour block of data is analyzed here. That 11 hour block was chosen because that was the longest available continuous block of data corresponding to very low wind conditions; during that 11 hour window the average wind speed was $1.9 \mathrm{~m} / \mathrm{s}$ and the maximum wind speed was $3.0 \mathrm{~m} / \mathrm{s}$. The wave data was sampled at $20 \mathrm{~Hz}$, and decimated to 10 $\mathrm{Hz}$ for the analysis shown here. The data at both buoys included some small gaps (see Fig. 6) that were filled with zeros. The wave spectra measured at both locations had energy concentrated in the frequency band $0.3 \mathrm{~Hz}<f<0.5$ $\mathrm{Hz}$, with a peak at $0.35 \mathrm{~Hz}$. This frequency band corresponds to wavelengths in the range $17 \mathrm{~m}>\lambda>6 \mathrm{~m}$ and group speeds in the range $2.6 \mathrm{~m} / \mathrm{s}>c_{g}>$ $1.5 \mathrm{~m} / \mathrm{s}$. During the measurement period, the wave field was highly directional, with wave energy propagating shoreward, from Yankee to Romeo. Waves in the excited frequency band take between approximately $70 \mathrm{~min}$ and $120 \mathrm{~min}$ to travel from Yankee to Romeo.

Before presenting results, we note that directional wave spectra at the two measurement locations have implications for the anticipated structure of the cross-correlation functions that we compute. If the cross correlation function is computed as $C_{A B}(t)=\left\langle\int_{0}^{T} d \tau \eta_{A}(\tau) \eta_{B}(\tau+t)\right\rangle$, and wave energy is propagating predominantly from $A$ to $B$, then deterministic structure is expected to be observed in $C_{A B}(t)$ for $t>0$, but not for $t<0$. That is the case here with $A$ and $B$ corresponding to Yankee and Romeo, respectively. Thus we expect, after coherent stacking is performed, to see a cross-correlation function in which almost all energy is at positive lag.

These comments lead us to expect deterministic structure in the stacked correlation function at positive lag between approximately $70 \mathrm{~min}$ and $120 \mathrm{~min}$, with maximum absolute amplitude at approximately $82 \mathrm{~min}$. Furthermore, the correlation function is expected to consist of a smooth frequency-modulated sweep with frequency increasing with increasing lag - like a one-sided version of Fig. 2, but with many more wiggles. Realizations of the correlation function were computed by sliding 210 min subsequences of the Romeo sea surface 
elevation time series relative to the Yankee time series. Adjacent subsequences were staggered by $30 \mathrm{~min}$. This gave 14 realizations (which are not fully independent of each other) of the cross-correlation function that were subsequently coherently averaged. Results are shown in Fig. 7. Unfortunately, there is no hint that the anticipated structure - or, indeed, any deterministic structure is emerging in the average cross-correlation function shown in Fig. 7. (Note that using a subsequence length of 210 min leads to a maximum lag of $105 \mathrm{~min}$, which is not quite long enough to capture the entire anticipated deterministic signal. We have also processed the data using a subsequence length of $264 \mathrm{~min}$, corresponding to a maximum lag of $132 \mathrm{~min}$. Those results are equally disappointing. The advantage of using the shorter subsequence length is that a few more realizations of the correlation function are produced.) Potential causes for the disappointing results described here are discussed below.

\section{Summary and discussion}

In this paper we have: 1) presented theoretical results that underlie random water wave interferometry; 2) tested the theoretical predictions using simulations in both open and closed systems; 3 ) tested the theoretical predictions using wave tank measurements; and 4) tested the theoretical predictions using ocean wave measurements.

Simulations were shown to be in very good agreement with theoretical predictions, but a caveat should be noted: Green's functions computed using Eq. 28) are causal only in the nondispersive limit. Note, however, that non-causal precursors in the Green's functions used to construct Figs. 1 and 2 are too small to be seen. In those figures non-causal precursors are negligibly small both because the precursors have negligible amplitude compared to the statistical uncertainty associated with our estimates of $C_{A B}(t)$, and because the temporal extent of the precursors is negligible compared to the temporal smearing associated with convolution with $D(t)$. In spite of the smallness of non-causal precursors in the Green's functions used to construct Figs. 1 and 2, it should be stated clearly 
that non-causal Green's functions are not physical, and that this shortcoming should be corrected. It is noteworthy that the Green's functions on which our closed domain simulations are based are causal and correspond to intermediate depth, i.e., dispersive, conditions. (Those simulations are shown in Fig. 3 and are also in very good agreement with theoretical predictions.) Thus dispersion need not lead to non-causal Green's functions. It has previously been recognized [46, 47] that, in general, the construction of causal water wave Green's functions requires special care. That topic is worthy of a renewed investigation in the context of water wave interferometry. A possibility is to assume weak dissipation and make use of a Kramers-Kronig relationship when constructing Green's functions.

More significant than our simulations, wavetank measurements were shown to be in good agreement (recall Fig. 5) with the fundamental result of water wave interferometry, Eq. (1).

The ocean measurements were not in agreement with theoretical predictions. As noted in the previous section, the correlation functions based on ocean measurements were expected to have a different character than the correlation functions based on simulations or wave tank measurements. Even with this caveat, our attempt to produce a band-limited estimate of a water wave Green's function using the SW06 data must be regarded as a failure. Several likely factors that contributed to this failure will now be discussed.

An important factor that likely contributed to the failure of our SW06 data 470 analysis is a shortcoming of the data. The relative drift of the spar buoys that made the ocean wave measurements was probably sufficiently large over the 11 hour data window that phase coherence between instruments was lost. The spar buoys were moored to anchors at $80 \mathrm{~m}$ depth. The expected maximum horizontal drift of each buoy relative to its mean position is about $90 \mathrm{~m}$ (N. 475 Williams, personal communication); relative drift between the two instruments is expected to be much less, but that quantity is more difficult to estimate. Temporal changes in buoy positions during the measurement period were likely due primarily to tidal currents. Note, in this regard, that the 11 hour data window 
is comparable to the M2 tidal period, 12.42 hours. The measured wave spectra anticipated structure. We have doubled the duration of the data analysis time window, from 11 to 22 hours, and repeated the analysis; like Fig. 7, the resulting stacked correlation function had none of the anticipated features that we 
have described. This might be explained by the higher average winds, $4.0 \mathrm{~m} / \mathrm{s}$, during the second 11 hours of the 22 hour window; the argument just presented suggests that wind effects over the second 11 hour block of data had a nonnegligible influence the wave field. We return now to the result shown in Fig. 7, corresponding to processing the first 11 hours only. In [5] and [13] it is estimated that convergence of the correlation function requires that the product of the minimum integration time $T$ and the coherent bandwidth exceeds a constant times $(k r)^{d-1}$ where $d=2$ for propagation in two space dimensions and $d=3$ for propagation in three space dimensions. It is typical in noise interferometry applications, including the work reported here, that the coherent bandwidth is approximately equal to the center frequency of the band of coherent energy. In the simulations shown in Figs. 1 and 2 the minimum integration time to obtain a rough estimate of the average correlation function was about 5 hours. Using that estimate and the differences in center frequency and $k r$, the formula just presented with $d=2$ predicts that for the SW06 data the minimum integration time required to yield a usable estimate of the average correlation function is about 30 days. This strongly suggests that the 11 hours of data that we used was far too short. But the validity of the assumption that $d=2$ might be questioned because of the strong directionality of the wave field, with waves propagating from Yankee to Romeo. For $d=1$ the formula just presented predicts that $T$ is independent of $k r$. If the $d=1$ limit of that formula is applicable, and if the SW06 measurements are described by $d=1$, the minimum integration time for the SW06 data can be estimated from the minimum integration time in our 1-d wavetank experiment - about 0.5 hour. That time interval corresponds to approximately 15 independent realizations of the correlation function. Assuming that 15 independent realizations of the correlation function are required for $d=1$, independent of $k r$, leads to the conclusion that, if $d=1$ for the SW06 data, about 20 hours of data is the minimum required for convergence. We expect that minimum required integration time for windless SW06-like conditions lies somewhere between the $d=1$ and $d=2$ estimates just given, leading to the conclusion that shortness of the SW06 data records used in our analysis was a 
significant factor contributing to the failure of our analysis.

A entirely different - and potentially more interesting - possible explanation for the failure of ocean-measurement-based estimates of correlation functions to reveal the predicted structure is inadequacy of the underlying theory. Specifi545 cally, the underlying theory that we have presented is based on linear theory, whose validity requires that $k a$, where $a$ is wave amplitude, is small. It is possible that finite amplitude effects that are neglected in the theory led to sufficiently large wave field changes between measurement locations that the predicted linear theory information contained in the cross-correlations was lost. In this regard, it is noteworthy that the most striking manifestation of nonlinear effects in nonbreaking ocean waves is the phase locking of short waves to longer waves, causing the short waves to propagate faster than is predicted by linear theory. (This accounts for a basic feature of ocean waves - wave crests are relatively sharp, while wave troughs are flat.) But this effect should not prevent one from obtaining stable estimates of correlation functions. Thus, we don't expect that this was the dominant cause of the failure of our ocean wave analysis. We note also that $k a$ was approximately 0.023 in our wavetank experiment, and 0.007 in the SW06 data that we used; this also suggests that breakdown of linear theory was not the principal cause of the failure of our SW06 analysis. More generally, the breakdown of random water wave interferometry due to nonlinear effects can - and should - be systematically investigated in a wave tank by performing several sets of experiments of the type described in section 4 , with varying $k a$.

\section{Acknowledgements}

We thank Brian Haus, Nathan Laxague and David Ortiz-Suslow for assistance with wave tank measurements. We thank Hans Graber, Rafael Ramos and Neil Williams for providing access to the ocean wave measurements analyzed here. This work was supported by the National Science Foundation, grant OCE1129860, and the Office of Naval Research, grant N000141210182. 
[1] S. M. Rytov, Y. A. Kravtsov, V. I. Tatarskii, Principles of Statistical Radiophysics. 3: Elements of Random Fields, Springer, New York, 1989.

[2] O. I. Lobkis, R. L. Weaver, On the emergence of the Green's function in the correlations of a diffuse field, J. Acoust. Soc. Am. 110 (2001) 3011-3017.

[3] K. Wapenaar, Retrieving the elastodynamic Green's function of an arbitrary inhomogeneous medium by cross correlation, Phys. Rev. Lett. 93 (2004) 254301.

[4] R. Snieder, Extracting the Green's function from the correlation of coda waves: A derivation based on stationary phase, Phys. Rev. E 69 (046610).

[5] R. L. Weaver, O. I. Lobkis, Fluctuations in diffuse field-field correlations and the emergence of the Green's function in open systems, J. Acoust. Soc. Am. 117 (2005) 3432-3439.

[6] K. G. Sabra, P. Roux, W. A. Kuperman, Emergence rate of the timedomain Green's function from the ambient noise cross-correlation function, J. Acoust. Soc. Am. 118 (2005) 3524-3531.

[7] O. A. Godin, Recovering the acoustic Green's function from ambient noise cross-correlation in an inhomogeneous moving medium, Phys. Rev. Lett. 97 (2006) 054301.

[8] O. A. Godin, Emergence of the acoustic Green's function from thermal noise, J. Acoust. Soc. Am 121 (2007) EL96-EL102.

[9] J. Garnier, J. Papanicolaou, Passive sensor imaging using cross correlations of noisy signals in a scattering medium, SIAM J. Imaging Sciences 2 (2009) 396-437.

[10] R. Weaver, B. Froment, M. Campillo, On the correlation of nonisotropically distributed ballistic scalar diffuse waves, J. Acoust. Soc. Am. 126 (2009) 1817-1826. 
[11] O. A. Godin, Cross-correlation function of acoustic fields generated by random high-frequency sources, J. Acoust. Soc. Am. 128 (2010) 600-610.

[12] M. G. Brown, Noise interferometry in the geometric limit in a multipathing environment, J. Acoust. Soc. Am. 130 (2011) EL173-EL179.

[13] N. A. Zabotin, O. A. Godin, Emergence of acoustic Green's functions from time averages of ambient noise, Acta Acustica united with Acustica 97 (2011) 44-53.

[14] O. A. Godin, Retrieval of Green's functions of elastic waves from thermal fluctuations of fluid-solid systems, J. Acoust. Soc. Am. 125 (2009) 19601970.

[15] R. Snieder, E. Safak, Extracting the building response using seismic interferometry; theory and application to the Millikan Library in pasadena, california, Bull. Seismol. Soc. Am. 96 (2006) 586-598.

[16] K. G. Sabra, E. S. Winkel, D. A. Bourgoyne, B. R. Elbing, S. L. Ceccio, M. Perlin, D. R. Dowling, On using cross-correlation of turbulent flowinduced ambient vibrations to estimate the structural impulse response. application to structural health monitoring, J. Acoust. Soc. Am. 121 (2007) 1987-2005.

[17] M. Campillo, A. Paul, Long-range correlations in the diffuse seismic coda, Science 299 (2003) 547-549.

[18] N. M. Shapiro, M. Campillo, L. Stehly, M. Ritzwoller, High resolution surface wave tomography from ambient seismic noise, Science 307 (2005) $1615-1618$.

${ }_{620}$ [19] Y. Yang, M. H. Ritzwoller, A. L. Levshin, N. M. Shapiro, Ambient noise Rayleigh wave tomography across europe, Geophys. J. Int. 168 (2007) 259274 . 
[20] R. Snieder, E. Larose, Extracting Earth's elastic wave response from noise measurements, Ann. Rev. Earth Planetary Sci. 41 (2013) 183-206.

[21] A. Gorbatov, E. Saygin, B. L. N. Kennett, Crustal properties from seismic station autocorrelograms, Geophys. J. Int. 192 (2013) 861-870.

[22] M. Campillo, P. Roux, Seismic imaging and monitoring with ambient noise correlations, in: Treatise of Geophysics by B. Romanowicz and A. Dziewonski, Elsevier, Amsterdam, 2014, pp. 256-271.

[23] T. L. Duvall Jr., S. M. Jeffferies, J. W. Harvey, M. A. Pomerantz, Timedistance helioseismology, Nature 362 (1993) 430-432.

[24] J. E. Rickett, J. F. Claerbout, Calculation of the Sun's impulse response by multi-dimensional spectral factorization, Solar Physics 192 (2000) 203-210.

[25] P. Roux, W. A. Kuperman, the NPAL Group, Extracting coherent wave fronts from acoustic ambient noise in the ocean, J. Acoust. Soc. Am. 116 (2004) 1995-2003.

[26] L. A. Brooks, P. Gerstoft, Ocean acoustic interferometry, J. Acoust. Soc. Am. 121 (2007) 3377-3385.

[27] O. A. Godin, N. A. Zabotin, V. V. Goncharov, Ocean tomography with acoustic daylight, Geophys. Res. Lett. 37 (2010) L13605. doi:10.1029/ 2010GL043623

[28] O. A. Godin, On the possibility of using acoustic reverberation for remote sensing of the ocean dynamics, Acoust. Phys. 58 (1) (2012) 129-138. doi: $10.1134 / \mathrm{S} 1063771012010101$

${ }_{645}[29]$ K. G. Sabra, S. Fried, W. A. Kuperman, M. Prior, On the coherent components of low-frequency ambient noise in the Indian Ocean, J. Acoust. Soc. Am. 133 (2013) EL20-EL25. 
[30] S. W. Lani, K. G. Sabra, W. S. Hodgkiss, W. A. Kuperman, P. Roux, Coherent processing of shipping noise for ocean monitoring, J. Acoust. Soc. Am. 133 (2013) EL108-EL113.

[31] M. Siderius, C. H. Harrison, M. B. Porter, A passive fathometer technique for imaging seabed layering using ambient noise, J. Acoust. Soc. Am. 120 (2006) 1315-1323.

[32] M. Siderius, H. Song, P. Gerstoft, W. S. Hodgkiss, P. Hursky, C. Harrison, Adaptive passive fathometer processing, J. Acoust. Soc. Am. 127 (2010) 2193-2200.

[33] M. G. Brown, O. A. Godin, N. J. Williams, N. A. Zabotin, L. Zabotina, G. J. Banker, Acoustic Green's function extraction from ambient noise in a coastal ocean environment, Geophys. Res. Lett. 41 (2014) 5555-5562.

[34] O. A. Godin, M. G. Brown, N. A. Zabotin, L. Zabotina, N. J. Williams, Passive acoustic measurement of flow velocity in the Straits of Florida, Geosci. Lett. 1 (2014) 16. doi:10.1186/s40562-014-0016-6.

[35] C. Yardim, P. Gerstoft, W. S. Hodgkiss, J. Traer, Compressive geoacoustic inversion using ambient noise, J. Acoust. Soc. Am. 135 (2014) 1245-1255.

[36] M. M. Haney, Infrasonic ambient noise interferometry from correlations

口 of microbaroms, Geophys. Res. Lett. 36 (2009) L19808. doi:10.1029/ 2009GL040179.

[37] J. T. Fricke, L. G. Evers, P. S. M. Smets, K. Wapenaar, D. G. Simons, Infrasonic interferometry applied to microbaroms observed at the Large Aperture Infrasound Array in the Netherlands, J. Geophys. Res. Atmospheres 119 (2014) 9654-9665.

[38] O. A. Godin, V. G. Irisov, M. I. Charnotskii, Passive acoustic measurements of wind velocity and sound speed in air, J. Acoust. Soc. Am. 135 (2) (2014) EL68-EL74. doi:10.1121/1.4862885. 
[45] H. C. Graber, E. A. Terray, M. A. Donelan, W. M. Drennan, J. C. van Leer, ASIS - A new Air-Sea Interaction Spar buoy: design and per-

[46] J. Falnes, On non-causal impulse response functions related to propagating water waves, Appl. Ocean Res. 17 (6) (1995) 379-389.

[47] C. J. Damaren, A causal transient water wave diffraction formulation, Appl. Ocean Res. 22 (5) (2000) 267-280.

[48] W. J. Plant, A relationship between wind stress and wave slope, J. Geophys. Res. Oceans 87 (C3) (1982) 1961-1967. 


\section{Figure captions}

Figure 1. Solid curve: cross-correlation function $C_{A B}(t)$ of $\eta\left(\mathbf{x}_{A}, t\right)$ and $\eta\left(\mathbf{x}_{B}, t\right)$ in an open weakly dispersive water wave system (assuming $\omega^{2}=g h k^{2}(1-$ $\left.(k h)^{2} / 3\right)$ with $\left.h=1 \mathrm{~m}\right)$, computed by coherently averaging approximately 5000 realizations of the correlation function based on 160 s blocks of simulated surface elevation. Dashed curve: $D(t) *\left[G_{\eta}\left(\mathbf{x}_{B} \mid \mathbf{x}_{A}, t\right)+G_{\eta}\left(\mathbf{x}_{A} \mid \mathbf{x}_{B},-t\right)\right]$, where $G_{\eta}\left(\mathbf{x}_{B} \mid \mathbf{x}_{A}, t\right)$ was computed using Eq. 28). Each curve is normalized to have maximum absolute amplitude equal to 1 .

${ }_{710}$ Figure 2. Solid curve: cross-correlation function $C_{A B}(t)$ of $\eta\left(\mathbf{x}_{A}, t\right)$ and $\eta\left(\mathbf{x}_{B}, t\right)$ in an open dispersive water wave system (assuming $\omega^{2}=g k$ ), computed by coherently averaging approximately 5000 realizations of the correlation function based on $160 \mathrm{~s}$ blocks of simulated surface elevation. Dashed curve: $D(t) *$ $\left[G_{\eta}\left(\mathbf{x}_{B} \mid \mathbf{x}_{A}, t\right)+G_{\eta}\left(\mathbf{x}_{A} \mid \mathbf{x}_{B},-t\right)\right]$, where $G_{\eta}\left(\mathbf{x}_{B} \mid \mathbf{x}_{A}, t\right)$ was computed using Eq. (28). Each curve is normalized to have maximum absolute amplitude equal to 1.

Figure 3. Solid blue curve: cross-correlation function $C_{A B}(t)$ for $t>0$ of $\eta\left(x_{A}, t\right)$ and $\eta\left(x_{B}, t\right)$, computed by coherently averaging 240 realizations of the correlation function based on 240 s blocks of simulated surface elevation in a wave tank. Dashed red curve: $D(t) * G_{\eta}\left(x_{B} \mid x_{A}, t\right)$, where $G_{\eta}\left(x_{B} \mid x_{A}, t\right)$ was computed using Eq. (29). Each curve is normalized to have maximum absolute amplitude equal to 1.

Figure 4. Blue curve: average measured spectrum $\left(\left|\bar{\eta}\left(x_{A}, \omega\right)\right|+\left|\bar{\eta}\left(x_{B}, \omega\right)\right|\right) / 2$ in a wave tank. Red vertical lines: theoretical spectrum $\sum_{l} \bar{d}\left(\omega_{l}\right)\left|\cos \left(k_{l} x_{A}\right)\right|$, where $\omega_{l}^{2}=g k_{l} \tanh k_{l} h$ and $k_{l}=l \pi / L_{x}$, based on an empirical smooth curve $\bar{d}(\omega)$. Each spectrum is normalized to have a peak value equal to 1 .

Figure 5. Solid blue curve: average cross-correlation function $\left(C_{A B}(t)+C_{A B}(-t)\right) / 2$ of $\eta\left(x_{A}, t\right)$ and $\eta\left(x_{B}, t\right)$ in a closed system, computed by coherently averaging 240 realizations of the correlation function based on $240 \mathrm{~s}$ blocks of measured surface elevation in a wave tank. Dashed red curve: $D(t) * G_{\eta}\left(x_{B} \mid x_{A}, t\right)$, where 
$G_{\eta}\left(x_{B} \mid x_{A}, t\right)$ was computed using Eq. 29 . The two curves are normalized so that both have the same integrated energy between 3 and $60 \mathrm{~s}$.

Figure 6. Concurrent eleven hour segments of time series of sea surface elevation measured at buoys Romeo and Yankee.

735 Figure 7. Cross-correlation function $C_{A B}(t)$ of $\eta\left(\mathbf{x}_{A}, t\right)$ and $\eta\left(\mathbf{x}_{B}, t\right)$ in an open system, computed by coherently averaging 16 realizations of the correlation function based on 210 min blocks of measured surface elevation in the ocean. $C_{A B}(t)$ is normalized to have maximum absolute amplitude equal to 1. 


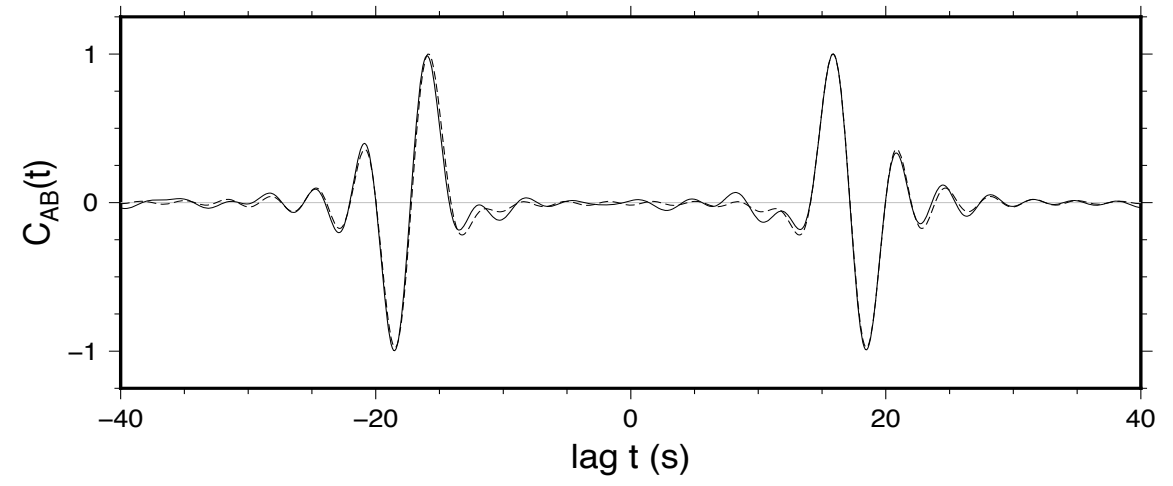

Figure 1: Solid curve: cross-correlation function $C_{A B}(t)$ of $\eta\left(\mathbf{x}_{A}, t\right)$ and $\eta\left(\mathbf{x}_{B}, t\right)$ in an open weakly dispersive water wave system (assuming $\omega^{2}=g h k^{2}\left(1-(k h)^{2} / 3\right)$ with $h=1 \mathrm{~m}$ ), computed by coherently averaging approximately 5000 realizations of the correlation function based on $160 \mathrm{~s}$ blocks of simulated surface elevation. Dashed curve: $D(t) *\left[G_{\eta}\left(\mathbf{x}_{B} \mid \mathbf{x}_{A}, t\right)+\right.$ $G_{\eta}\left(\mathbf{x}_{A} \mid \mathbf{x}_{B},-t\right)$ ], where $G_{\eta}\left(\mathbf{x}_{B} \mid \mathbf{x}_{A}, t\right)$ was computed using Eq. 28). Each curve is normalized to have maximum absolute amplitude equal to 1. 


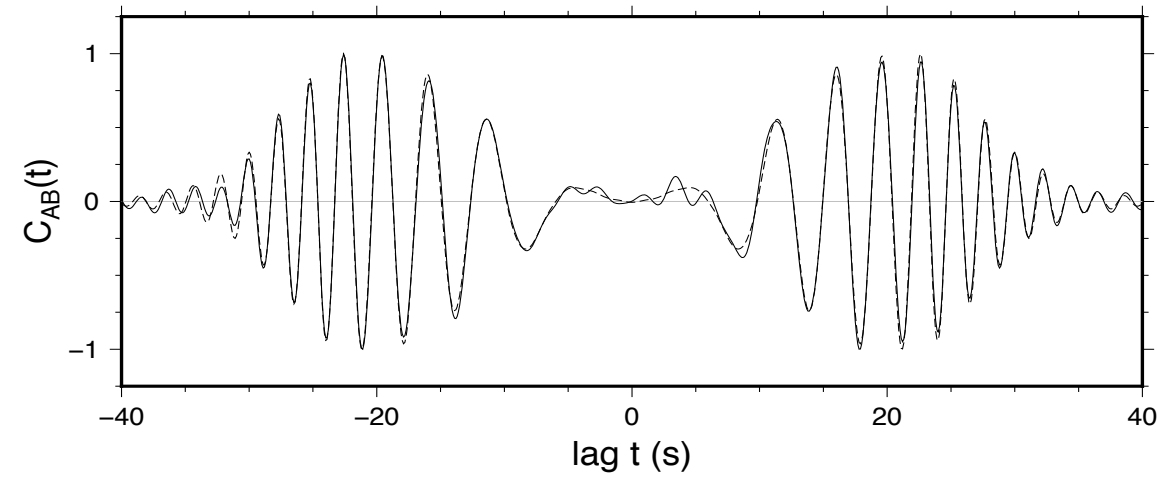

Figure 2: Solid curve: cross-correlation function $C_{A B}(t)$ of $\eta\left(\mathbf{x}_{A}, t\right)$ and $\eta\left(\mathbf{x}_{B}, t\right)$ in an open dispersive water wave system (assuming $\omega^{2}=g k$ ), computed by coherently averaging approximately 5000 realizations of the correlation function based on $160 \mathrm{~s}$ blocks of simulated surface elevation. Dashed curve: $D(t) *\left[G_{\eta}\left(\mathbf{x}_{B} \mid \mathbf{x}_{A}, t\right)+G_{\eta}\left(\mathbf{x}_{A} \mid \mathbf{x}_{B},-t\right)\right]$, where $G_{\eta}\left(\mathbf{x}_{B} \mid \mathbf{x}_{A}, t\right)$ was computed using Eq. 28. Each curve is normalized to have maximum absolute amplitude equal to 1.

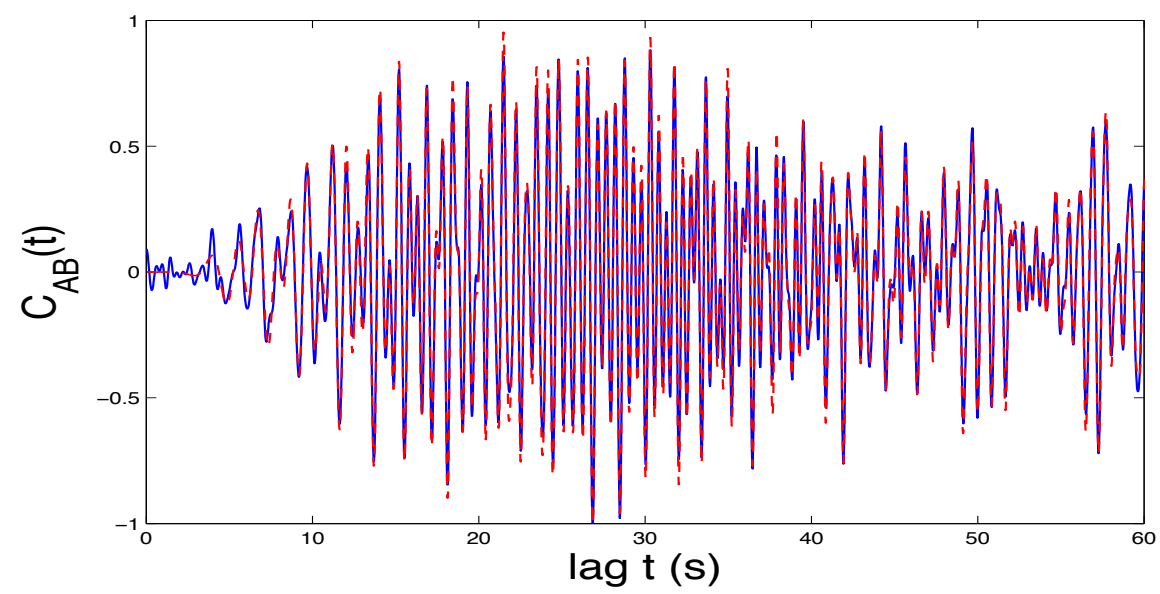

Figure 3: Solid blue curve: cross-correlation function $C_{A B}(t)$ for $t>0$ of $\eta\left(x_{A}, t\right)$ and $\eta\left(x_{B}, t\right)$, computed by coherently averaging 240 realizations of the correlation function based on $240 \mathrm{~s}$ blocks of simulated surface elevation in a wave tank. Dashed red curve: $D(t) * G_{\eta}\left(x_{B} \mid x_{A}, t\right)$, where $G_{\eta}\left(x_{B} \mid x_{A}, t\right)$ was computed using Eq. 29. Each curve is normalized to have maximum absolute amplitude equal to 1 . 


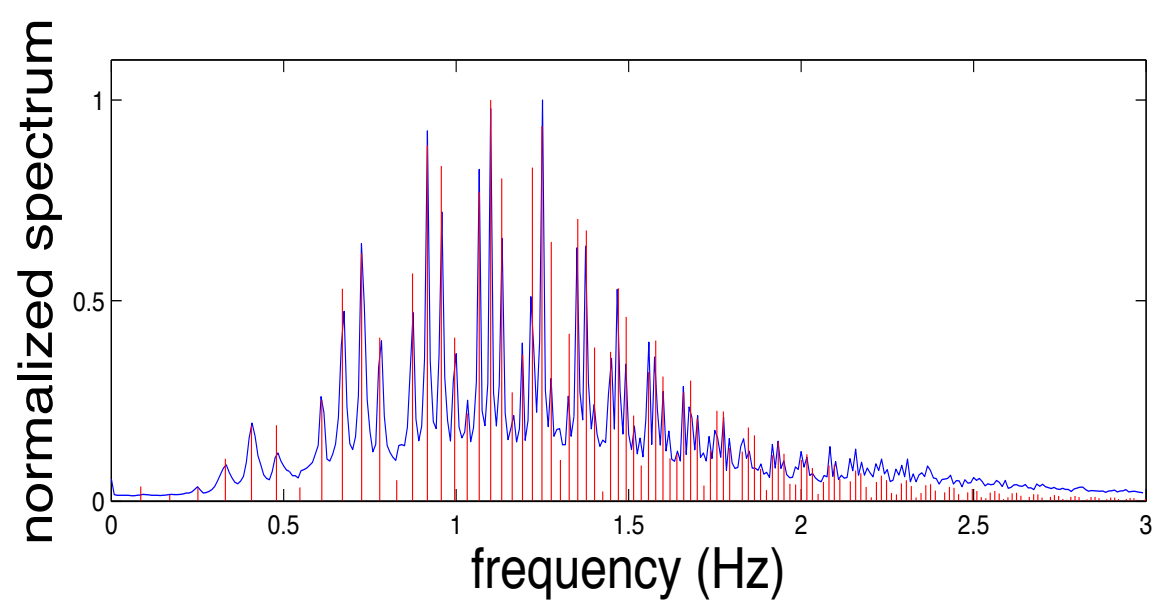

Figure 4: Blue curve: average measured spectrum $\left(\left|\bar{\eta}\left(x_{A}, \omega\right)\right|+\left|\bar{\eta}\left(x_{B}, \omega\right)\right|\right) / 2$ in a wave tank. Red vertical lines: theoretical spectrum $\sum_{l} \bar{d}\left(\omega_{l}\right)\left|\cos \left(k_{l} x_{A}\right)\right|$, where $\omega_{l}^{2}=g k_{l} \tanh k_{l} h$ and $k_{l}=l \pi / L_{x}$, based on an empirical smooth curve $\bar{d}(\omega)$. Each spectrum is normalized to have a peak value equal to 1 .

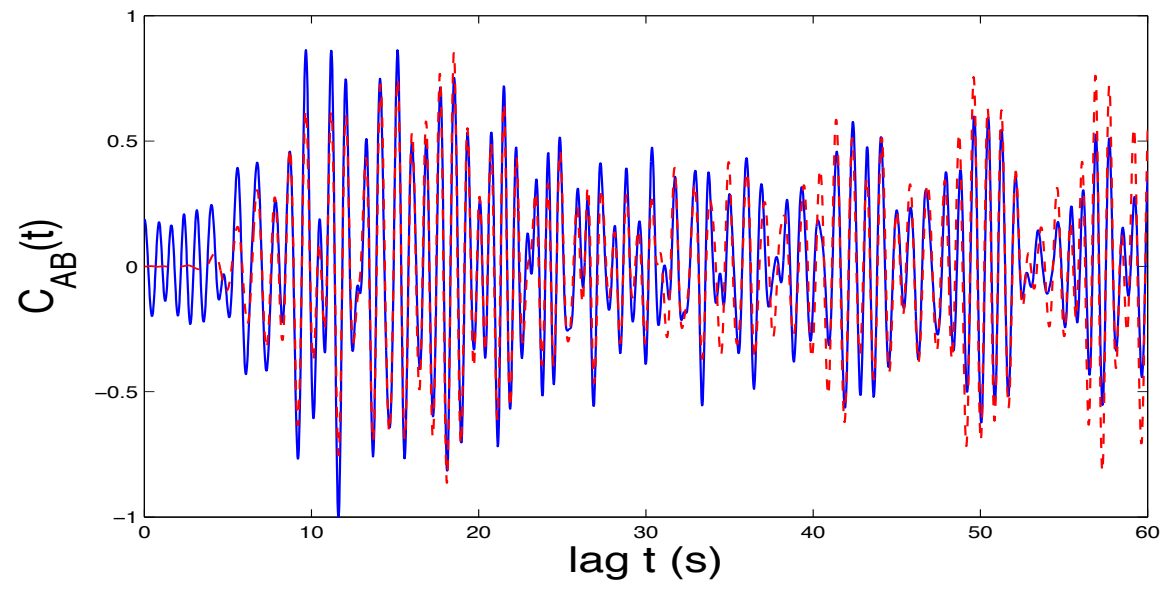

Figure 5: Solid blue curve: average cross-correlation function $\left(C_{A B}(t)+C_{A B}(-t)\right) / 2$ of $\eta\left(x_{A}, t\right)$ and $\eta\left(x_{B}, t\right)$ in a closed system, computed by coherently averaging 240 realizations of the correlation function based on $240 \mathrm{~s}$ blocks of measured surface elevation in a wave tank. Dashed red curve: $D(t) * G_{\eta}\left(x_{B} \mid x_{A}, t\right)$, where $G_{\eta}\left(x_{B} \mid x_{A}, t\right)$ was computed using Eq. 29 . The two curves are normalized so that both have the same integrated energy between 3 and $60 \mathrm{~s}$. 


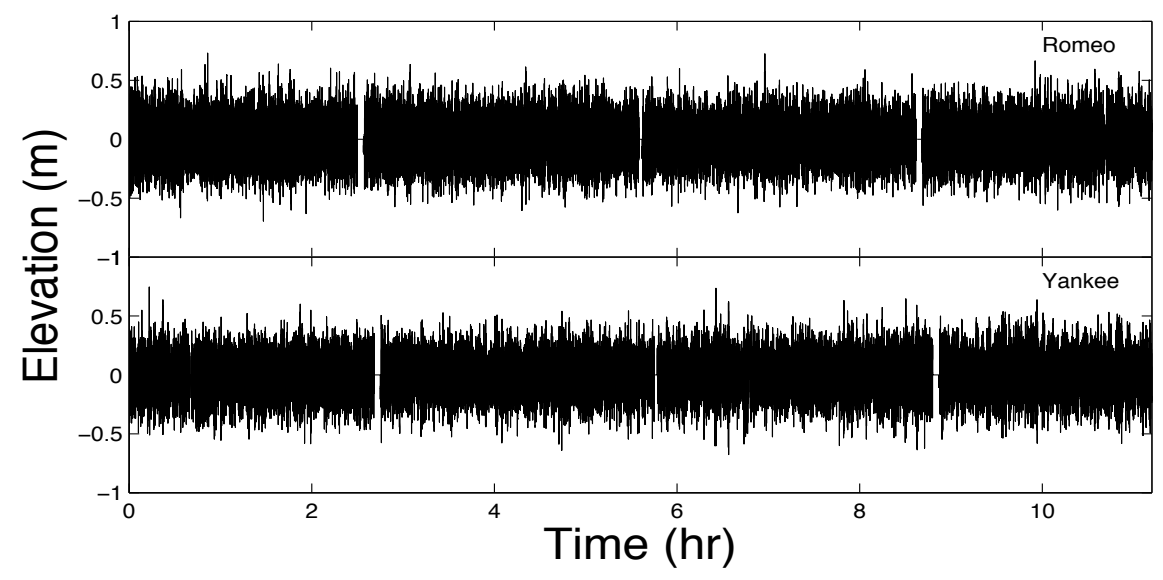

Figure 6: Concurrent eleven hour segments of time series of sea surface elevation measured at buoys Romeo and Yankee.

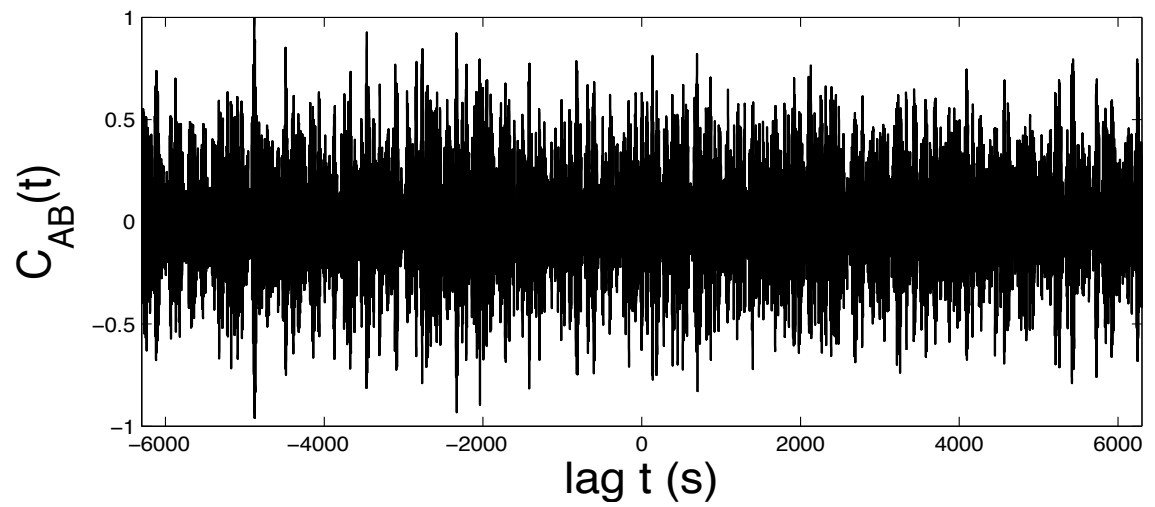

Figure 7: Cross-correlation function $C_{A B}(t)$ of $\eta\left(\mathbf{x}_{A}, t\right)$ and $\eta\left(\mathbf{x}_{B}, t\right)$ in an open system, computed by coherently averaging 16 realizations of the correlation function based on $210 \mathrm{~min}$ blocks of measured surface elevation in the ocean. $C_{A B}(t)$ is normalized to have maximum absolute amplitude equal to 1. 